\title{
Asymptotic freedom with discrete spin variables?
}

\author{
Peter Hasenfratz and Ferenc Niedermayer* ${ }^{\dagger}$ \\ Institute for Theoretical Physics, University of Bern, \\ Sidlerstrasse 5, CH-3012 Bern, Switzerland \\ E-mail: hasenfra@itp.unibe.ch niederma@itp.unibe.chi
}

ABSTRACT: We study the critical behaviour of the 2d dodecahedron spin model and investigate the conjecture that the discrete model describes the same continuum theory as the $\mathrm{O}(3)$ non-linear sigma model. In particular, we found that the anisotropy of the magnetization $A(z)$ measured in a fixed physical volume decreases with increasing correlation length, at least up to $\xi \approx 1000$.

One of the most studied quantum field theories is the $2 \mathrm{~d}$ non-linear sigma model. The lattice regularized version for the $\mathrm{O}(3)$ case is given by the action

$$
\mathcal{A}(S)=-\frac{1}{g} \sum_{x, \mu} S(x) S(x+\hat{\mu})
$$

where $S(x)$ is a unit $\mathrm{O}(3)$ vector, $S^{2}(x)=1$. The model is widely believed to be asymptotically free $(\mathrm{AF})$ and to have a dynamically generated mass gap.

Here we discuss the analogous model when the spin variables are allowed to take only discrete values pointing towards the vertices of a Platonic regular solid, e.g. icosahedron (I) or dodecahedron (D). Accordingly, the symmetry of the discrete model is reduced to a discrete subgroup $\mathcal{D}$ of $\mathrm{O}(3)$ (in the actual case, to the icosahedral group with 60 elements). At small $g$ (low temperature) the discrete symmetry is spontaneously broken while at large $g$ the system is in the symmetric phase under $\mathcal{D}$. Numerical evidence shows that there is a second order phase transition at some $g=g_{c}>0$.

A few years ago Patrascioiu and Seiler [i]1] observed that within their statistical errors the physical results for the dodecahedron model for $g \searrow g_{c}$ are consistent with those of the $\mathrm{O}(3)$ model and conjectured that the two models are equivalent in the continuum limit. The authors saw this observation as an argument supporting their unorthodox view that the $\mathrm{O}(3)$ model is not AF.

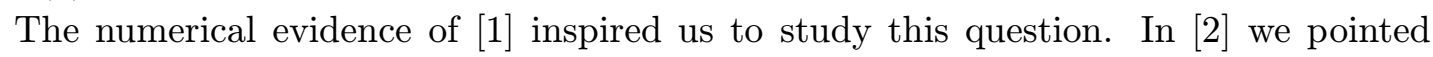
out that the equivalence is not in contradiction with the $\mathrm{O}(3)$ model being AF. Of course,

${ }^{*}$ Speaker.

${ }^{\dagger}$ On leave from Eötvös University, Budapest 


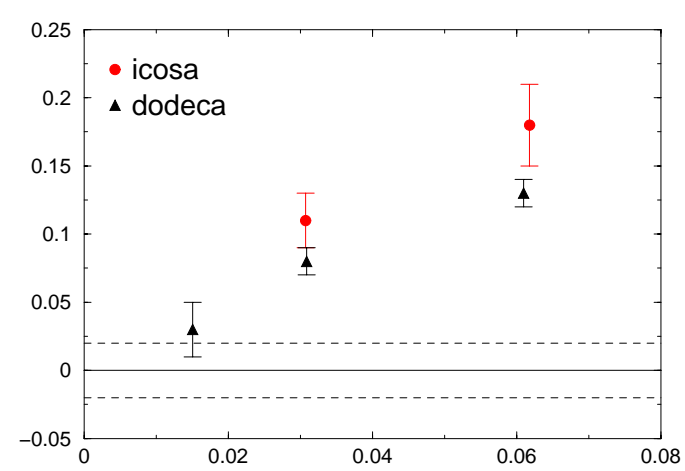

Figure 1: Deviation of $g_{R}(z)$ from the $\mathrm{O}(3)$ result vs. $1 / \xi(L)$.

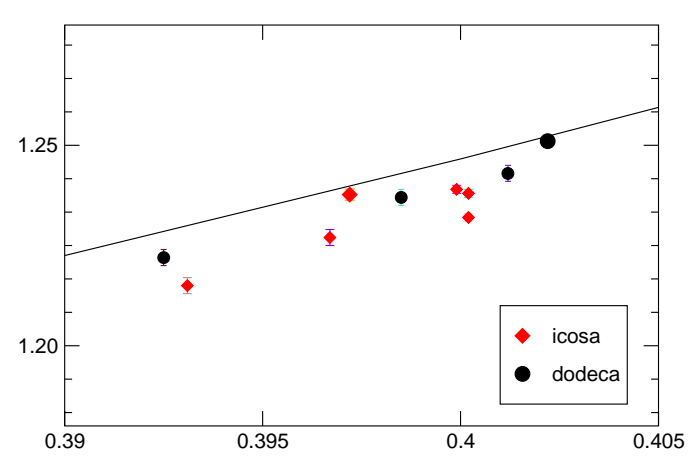

Figure 2: The FSS function $\xi(2 L) / \xi(L)$ vs $1 / z=\xi(L) / L$.

for the discrete model this is not a statement about the bare coupling (which goes to a non-zero constant in the continuum limit) but about some physical running coupling,

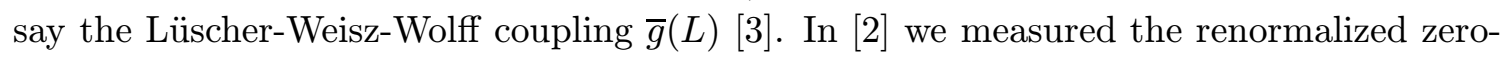
momentum 4-point coupling $g_{R}$ and have found that the I and D models approach the $\mathrm{O}(3)$ model with increasing $\xi$. In that work we went up to correlation length ${ }^{1} \xi \approx 300$ and reached precision of $\mathrm{O}(0.1 \%)$.

In [2] we also compared the finite size scaling (FSS) function of the LWW coupling in the discrete models to that of the $\mathrm{O}(3)$ model, with a similar conclusion.

Recently Caracciolo et al. [4-4] the $\mathrm{O}(3)$ action to break the original $\mathrm{O}(3)$ symmetry down to $\mathcal{D}$ is a relevant operator and concluded that the equivalence cannot hold. They suggested that the numerical evidence is misleading and the discrete model should belong to a different universality class, although they expected this to show up only at $\xi \gtrsim 200$. At this conference they have presented measurements of the FSS function and concluded that the discrete model starts to depart from the $\mathrm{O}(3)$, although at much larger correlation length, $\xi \approx 10^{5}[\overline{5}]$. We shall reflect on their arguments in the concluding section.

For the discrete models we have measured $g_{R}(z)$, the renormalized coupling at a fixed finite physical volume, $z=L / \xi(L)$, where $\xi(L)$ is the "second-moment correlation length". Fig. 'ili shows the deviation of $g_{R}(z)$ from $\mathrm{O}(3)$ at $z=2.32$ as a function of $1 / \xi(L)$, both for

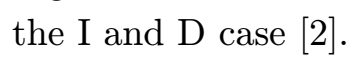

The FSS function $\xi(2 L) / \xi(L)$ for the icosahedron and dodecahedron models [2] is shown in fig.2. Larger symbols refer to larger correlation length, the largest one being

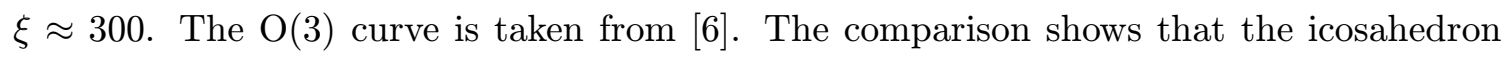
and dodecahedron models approach the $\mathrm{O}(3)$ result with increasing correlation length. (The results for tetrahedron, octahedron and cube - not shown here - depart from it.)

The restoration of the $\mathrm{O}(3)$ symmetry can be tested, however, in a more direct way by measuring the direction of average magnetization in a fixed physical volume and checking whether its distribution becomes uniform in the continuum limit or still prefers the original

\footnotetext{
${ }^{1}$ Here and below by $\xi$ we denote the infinite volume correlation length. When not measured directly it is calculated from the FSS function of [i6n".
} 
discrete directions. To do this we introduce the quantity

$$
I_{n}(S)=c\left\{\frac{1}{N} \sum_{R}(R S)^{n}-\frac{1}{N^{\prime}} \sum_{R^{\prime}}\left(R^{\prime} S\right)^{n}\right\},
$$

where $S$ is an arbitrary $\mathrm{O}(3)$ vector, $R$ runs over the set of vectors of the actual discrete model, $\left\{R_{i}, i=1, \ldots, N\right\}$ while $R^{\prime}$ runs over the analogous set of the dual regular solid (i.e. the unit vectors pointing towards the face centers of the original regular solid). For example, in the case of the dodecahedron $N=20$ and the dual model is the icosahedron with $N^{\prime}=12$. The value of $n$ is chosen to be the smallest integer for which $I_{n}(S)$ is not identically zero. For the dodecahedron this is $n=6$. Obviously, the integral of $I_{n}(S)$ over $S$ is zero, and $I_{n}(S)$ preserves the discrete symmetry $\mathcal{D}$. We fix the normalization by the condition $I_{n}(R)=1$ (where $R$ is one of the discrete directions). Up to the normalization constant our $I_{n}(S)$ coincides with the one introduced by Caracciolo et al. [4in] ${ }^{2}$

Consider now a fixed physical volume given by $z=L / \xi(L)$ and define the anisotropy

$$
A(z)=\frac{\left\langle I_{6}(M)\right\rangle}{\left\langle M^{2}\right\rangle^{3}}, \quad \text { where } \quad M=\sum_{x} R(x) .
$$

This quantity is a direct measure of the possible restoration of the $\mathrm{O}(3)$ symmetry in the continuum limit. Fig. 3 shows the anisotropy $A(z)$ for the dodecahedron at $z=1.888,1.668$ and 1.525 against $1 / \xi$. As the plot shows, $A(z)$ decreases with increasing $\xi$, at least up to our last value, $\xi \approx 1000$. This means that the dodecahedron model approaches the $\mathrm{O}(3)$ model. If the decrease persists and $\lim _{\xi \rightarrow \infty} A(z ; \xi)=0$ then they are equivalent in the continuum limit.

Similarly to Caracciolo et al. [4]

$$
\mathcal{A}(S)=-\frac{1}{g} \sum_{x, \mu} S(x) S(x+\hat{\mu})-h \sum_{x} I_{n}(S(x)),
$$

which interpolates between the $\mathrm{O}(3)$ model $(h=0)$ and the discrete model $(h=\infty)$. Here one expects a critical line $g_{c}(h)$ connecting $g_{c}(0)=0$ with the critical point $g_{c}(\infty)$ of the discrete model. For $g<g_{c}(h)$ the discrete symmetry $\mathcal{D}$ is spontaneously broken, while for $g>g_{c}(h)$ the system is in its symmetric phase. Since (by the standard wisdom) the correlation length at $g>0, h=0$ is finite, one expects that for $h \ll m^{2}(g)$ (where $m(g)=1 / \xi(g)$ is the inverse of the $\mathrm{O}(3)$ correlation length) no phase transition can occur. This is because the effect of the corresponding term on the independently fluctuating regions of size $\xi(g)$ is negligible. (The situation here is in sharp contrast to the $\mathrm{O}(2)$ case where in the massless phase an arbitrarily small external field can have a drastic effect on the system in an infinite volume.)

One can study the RG flow in the $(g, h)$ plane. The scenario advocated by Caracciolo et al. [i-i means that the RG flow along the critical line $g_{c}(h)$ is upwards, towards the $h=\infty$ point, while if the two models are in the same universality class the flow should be directed

\footnotetext{
${ }^{2}$ Although this is not directly seen since they used a concrete representation for the discrete spin vectors.
} 


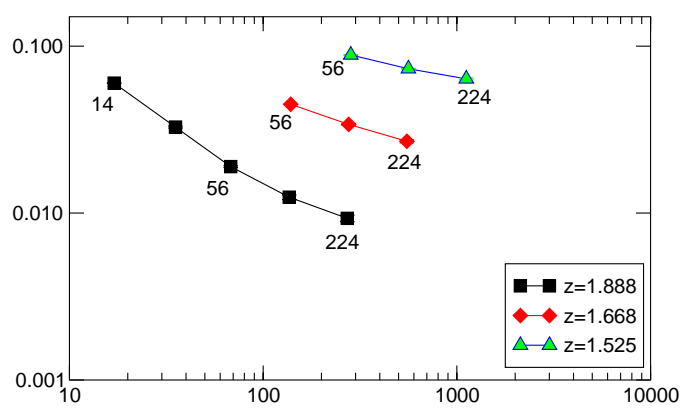

Figure 3: The anisotropy $A(z)$ vs. $\xi$.

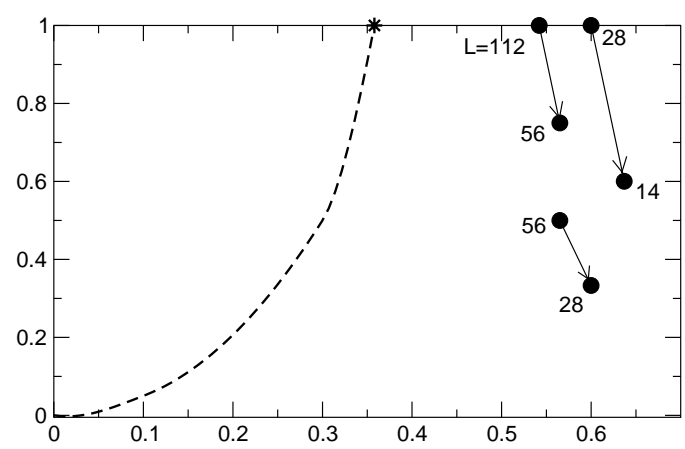

Figure 4: RG flow in the $(g, h)$ plane. On the vertical axis the values of $h /(h+1)$ are plotted.

downwards, towards $g=h=0$. A decisive answer would be provided by measuring the flow in the vicinity of the critical line. For example, consider a point $(g, h)$ corresponding to a fixed physical volume, $z=L / \xi(L)$ and with anisotropy $A(z)$. To determine the direction of the flow one has to find a point $\left(g^{\prime}, h^{\prime}\right)$ and size $L^{\prime}$ such that $\xi^{\prime}\left(L^{\prime}\right)=s \xi(L), L^{\prime}=s L$ (i.e. $z^{\prime}=z$ ) and $A^{\prime}(z)=A(z)$, with a scale factor $s<1$. At present we have data only for a few points, with $s=1 / 2$, at $z \approx 1.9$. The matching pairs $(g, h) \rightarrow\left(g^{\prime}, h^{\prime}\right)$ are shown in fig. 题 where the values of $L$ and $L^{\prime}$ are also indicated. Accordingly, our largest correlation length was $\xi(L) \approx 60$ which corresponds to $\xi \approx 130$. (The dashed line representing $g_{c}(h)$ is

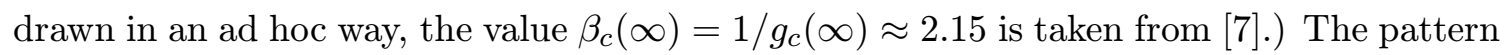
in fig. 育i suggests a downwards flow, but one would need points at larger correlation length to get a convincing answer.

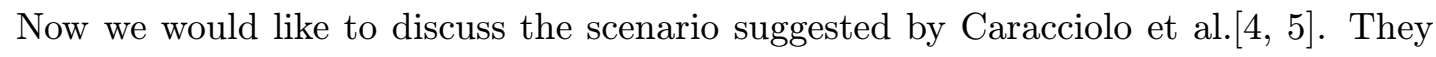
expand the correlation functions (defined in a finite $L \times L$ volume) in double power series in $g$ and $h$. Although the coefficients of this expansion diverge as $L \rightarrow \infty$ the functions appearing in the corresponding RG equation stay finite in this limit. The assumption they implicitly make is that the RG equation obtained remains valid if both $g$ and $h$ are small enough independently. Below we shall argue that the RG flow obtained by PT is valid only in a small wedge around the $h=0$ axis, for $h \ll 1 / R^{2}$ where $R=\min \{\xi(g), L\}$. In the extreme case of $g=0, L=$ finite (and for simplicity with the usual magnetic field coupled to $\left.S_{z}(x)\right)$ one has $\left\langle S_{z}\right\rangle=1 / \tanh \left(h L^{2}\right)-1 /\left(h L^{2}\right)$. For fixed $L$ this has a power series expansion in $h$ with infrared divergent coefficients. This expansion becomes, however, invalid for $h L^{2} \gg 1$ where the true answer is $\left\langle S_{z}\right\rangle \approx 1-1 /\left(h L^{2}\right)$. A similar behaviour is expected for finite $g$, with $L$ replaced by $\min \{\xi(g), L\}$. The reason for the existence of these two regimes is obvious: the system behaves differently depending on whether the external field is strong enough to keep the (independently fluctuating) regions of size $R$ in the preferred direction or not.

The argument given above is also valid for an $h I_{n}(S)$ term in the action. There is, however, an important difference: in the latter case the remnant discrete symmetry is spontaneously broken at $h>h_{c}(g)$. This breaking is associated with tunneling between different minima of $-h I_{n}(S)$, i.e. the dodecahedron directions. It is quite obvious that this 
tunneling cannot be described by PT: one can replace $-I_{n}(S)$ by a function which has one single minimum in one of the preferred discrete directions and has the same perturbative expansion around this minimum as $-I_{n}(S)$. PT will not notice any difference between the two cases while in the second case no discrete symmetry is left hence no phase transition will occur by increasing $h$. Although these are only speculative arguments, we think they could be made more rigorous.

As mentioned above, in [5] $\left[\begin{array}{l}0 \\ 0\end{array}\right]$ the authors also found that the FSS function for the dodecahedron starts to show a deviation from the $\mathrm{O}(3)$ model at huge correlation length, $\xi \approx 10^{5}$. Our criticism is the same as given by Patrascioiu and Seiler [욤]: 1) for $\xi \approx 10^{5}$ and $L=150$ the physical size of the system is practically zero and this small distances are dominated still by cutoff effects, 2) at such huge estimated $\xi$ one cannot be sure that the system is in the symmetric phase.

Summarizing, we think that the spontaneous breaking of the discrete symmetry is a non-perturbative critical phenomenon, even in the vicinity of the $g=h=0$ point. Concerning the numerical evidence - in particular the anisotropy measurements up to $\xi \approx 1000$ and the fact that in this range of $\xi$ Caracciolo et al. do not see either a deviation in the FSS behaviour - indicate that the dodecahedron (and the icosahedron) models describe the same continuum theory as the $\mathrm{O}(3)$ model. The question, however, deserves further numerical study.

The authors thank János Balog, Sergio Caracciolo, Martin Lüscher, Andrea Montanari, Andrea Pelissetto and Peter Weisz for useful discussions and correspondence. This work is supported in part by Schweizerische Nationalfonds and by the European Community's Human Potential Programme under HPRN-CT-2000-00145 Hadrons/Lattice QCD, BBW Nr. 990143.

\section{References}

[1] A. Patrascioiu and E. Seiler, Phys. Lett. B $\mathbf{4} 30$ (1998) 3144, hep-lat/9706011!

[2] P. Hasenfratz and F. Niedermayer, Nucl. Phys. B $\mathbf{5 9 6}(2001)$ 481, hep-1at/0006021;

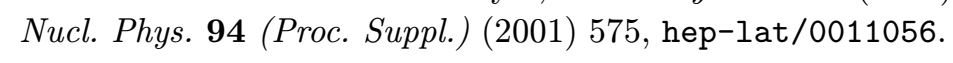

[3] M. Lüscher, P. Weisz and U. Wolff, 'Nucl. Phys. B-359 (1991) 22 1 i.

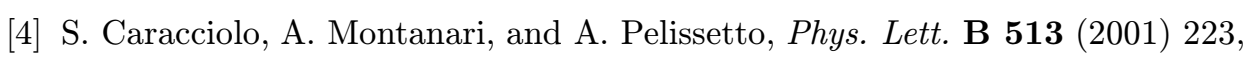
hep-iat/0103017.

[5] S. Caracciolo, A. Montanari, and A. Pelissetto, This conference, hep-lat/o110221.

[6] S. Caracciolo, R. G. Edwards, A. Pelissetto, and A. D. Sokal, 'Phys. Rev. Lett. 75 (1995)

L 1891', hep-lat/9411009r S. Caracciolo, R. G. Edwards, S. J. Ferreira, A. Pelissetto, and A. D. Sokal, Phys. Rev. Lett. 74 (1995) 2969 , hep-1at/9409004.

[7] A. Patrascioiu, J. L. Richard and E. Seiler, 'P

[8] A. Patrascioiu and E. Seiler, hep-latt/0110213. 